\title{
Tarifdelegierten-Tag FMH - Staatsmedizin versus Tarifautonomie
}
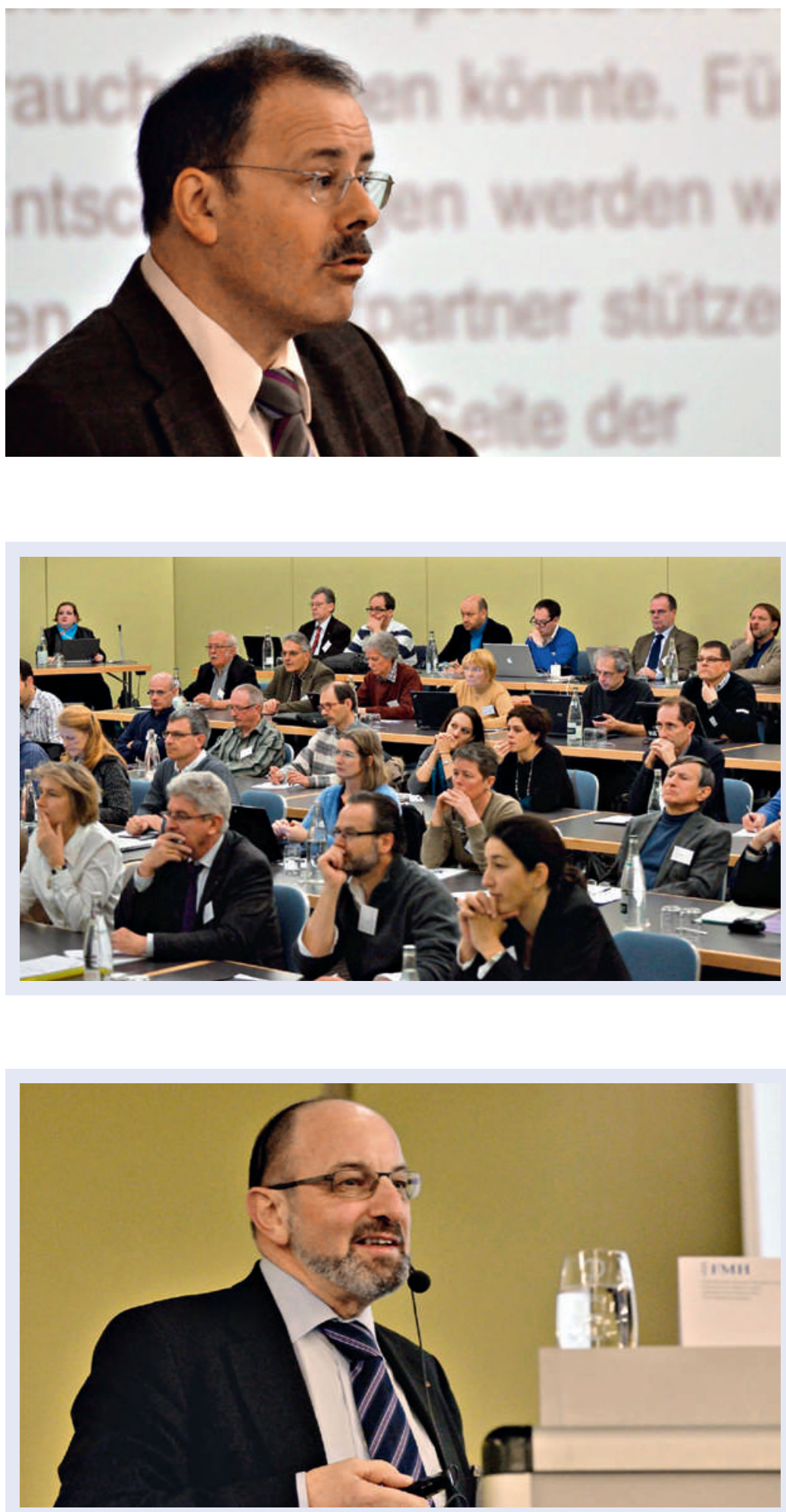

Pünktlich zum (astronomischen) Frühlingsanfang sind wieder über 80 Tarifdelegierte und Gäste auf Einladung der FMH zum Tarifdelegierten-Tag zusammengekommen, um gemeinsam aktuelle Tariffragen zu diskutieren. Der Präsident der FMH Dr. Jürg Schlup eröffnete die Veranstaltung und begrüsste die Teilnehmerinnen und Teilnehmer.

Das Ressort FMH Ambulante Tarife und Verträge Schweiz wählte als zentrales Thema «Staatsmedizin versus Tarifautonomie». Im Rahmen von Referaten und Workshops konnten sich die Delegierten intensiv mit Themen auseinandersetzen, die direkt oder indirekt mit dem Hauptthema verknüpft sind. Die Tarifautonomie im Gesundheitswesen funktioniert nur, wenn die Partner gemeinsam effiziente und wirksame Lösungen finden. Der Grundsatz für die Tarifautonomie muss dabei heissen: So viel Staat wie nötig - so viel Autonomie wie möglich! Die Referentinnen und Referenten waren eingeladen, hierzu Stellung zu nehmen und ihre Position dem Plenum zu erörtern.

Die aktuelle Situation im Tarifbereich:

\section{Dr. Ernst Gähler:}

«Die Revision der Tarifstruktur TARMED kommt seit dem Start 2010 mit dem Projekt TARVISION der FMH und seit 2012 mit der tripartiten Zusammenarbeit von FMH, $\mathrm{H}+$ und der MTK gut voran. Die Version TARMED 2.0 sollte unabhängig der Entscheide rund ums Kapitel $40 \mathrm{im}$ Herbst 2013 vorliegen. Im Bereich des Praxislabors hat die FMH dem BAG einen Vorschlag eingereicht, der den Vorgaben aus dem Monitoring des BAG entspricht. Die aktuellen Lösungsvorschläge des BAG und von Experten von FAMH und SULM gefährden die Existenz des Praxislabors. Die Verhandlungen zum margenfreien Abgeltungsmodell sind aktuell sistiert.» 

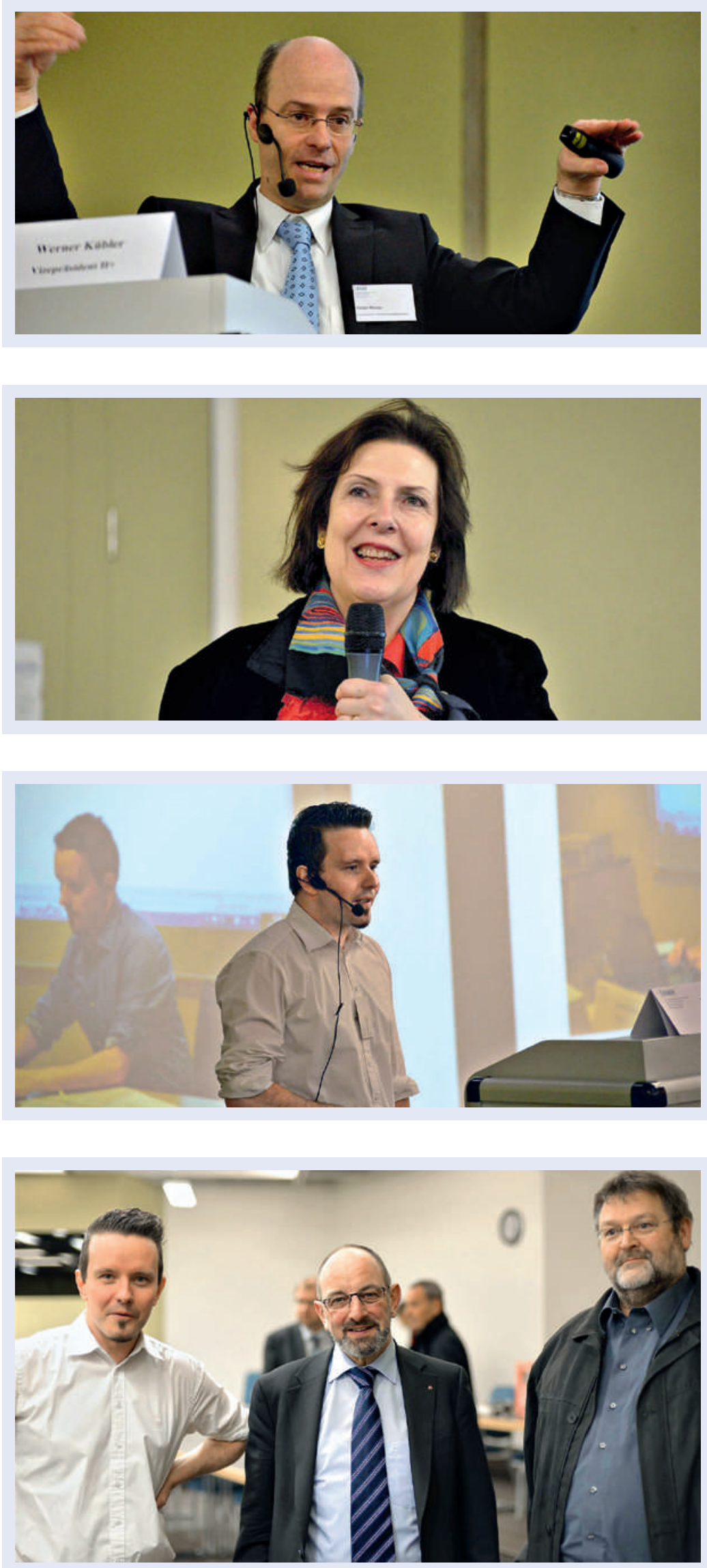

$\mathrm{H}+$ setzt sich für die Tarifautonomie ein: Was sind die Bedingungen für eine funktionierende und partnerschaftliche Tarifautonomie?

\section{Dr. Werner Kübler:}

«Tarifautonomie bedeutet für uns in erster Linie, dass sich die Tarifpartner gegenseitig respektieren und gewillt sind, für Lösungen Kompromisse einzugehen. Nur so können sich Tarifstrukturen den jeweils aktuellen medizinischen Leistungen und Kosten anpassen. $\mathrm{H}+$ setzt sich deshalb für Mehrheitsentscheide statt Vetorechte ein. Langfristig ist es viel schädlicher für die Tarifpartner, wenn Tarifstrukturen wegen gegenseitiger Blockaden veraltet bleiben, als bei einem Entscheid auch mal zu unterliegen.»

\section{Christine Egerszegi}

Die Ständerätin vermittelte den Teilnehmern die politische Perspektive auf das Veranstaltungsthema «Staatsmedizin versus Tarifautonomie». An Themen wie Einheitskasse, Hausarztinitiative und Zulassungsstop diskutierte Frau Egerszegi Anforderungen und Konsequenzen von Regulierungsmassnahmen im Gesundheitsbereich durch die Politik.

In ihrem Referat forderte sie die Ärzteschaft auf, mit den anderen Akteuren des Gesundheitswesens in einen Dialog zu treten und sich in diesem gemeinsamen Diskurs für die eigenen Ideen einzusetzen.

\section{Warum muss die «Tarifmaschine» wieder besser laufen?}

Roger Scherrer:

«Die FMH setzt sich klar für den Erhalt der Tarifautonomie ein. Dazu ist es jedoch unabdingbar, dass die Tarifpartner künttig schneller mit tarifarischen Lösungen auf neue Herausforderungen im medizinischen Alltag reagieren können. Die ganze ‘Tarifmaschine> muss künftig wieder effizienter produzieren. Mit dem FMH-internen Revisionsprojekt TARVISION leistet die FMH dazu einen wichtigen Beitrag. Zusammen mit den Partnern $\mathrm{H}+$ und MTK treibt sie die TARMED-Revision intensiv voran.»
Workshop 1: Gesundheitsversorgung $20 \%$ günstiger. Was trägt die Ärzteschaft bei?

Gemäss der kürzlich veröffentlichten Gesamtstrategie «Gesundheit 2020" strebt der Bund innerhalb weniger Jahre eine noch bessere Gesundheitsversorgung für massiv weniger Geld an. Für Sie ein realistisches Ziel?

\section{Anton Prantl, Dr. Ernst Gähler, Roger Scherrer:}

«Ungenannte Gesundheitsexperten gehen gemäss Bericht davon aus, dass zur Finanzierung rund 20 Prozent Effizienzreserven ausgeschöpft werden können.

Der Workshop zeigte: Die Ärzteschaft leistet bereits seit Jahren einen Beitrag zu einem finanzierbaren Gesundheitswesen in der geforderten Grössenordnung und bietet auch in Zukunft Hand für nachhaltige Lösungen. Nicht sachgerechte Eingriffe in

Tarifstrukturen und noch mehr Kontrolladministration können die Probleme aber nicht nachhaltig lösen und stehen im Widerspruch zu der im Bericht geforderten Kostentransparenz und zu den gesetzlichen Vorgaben.» 

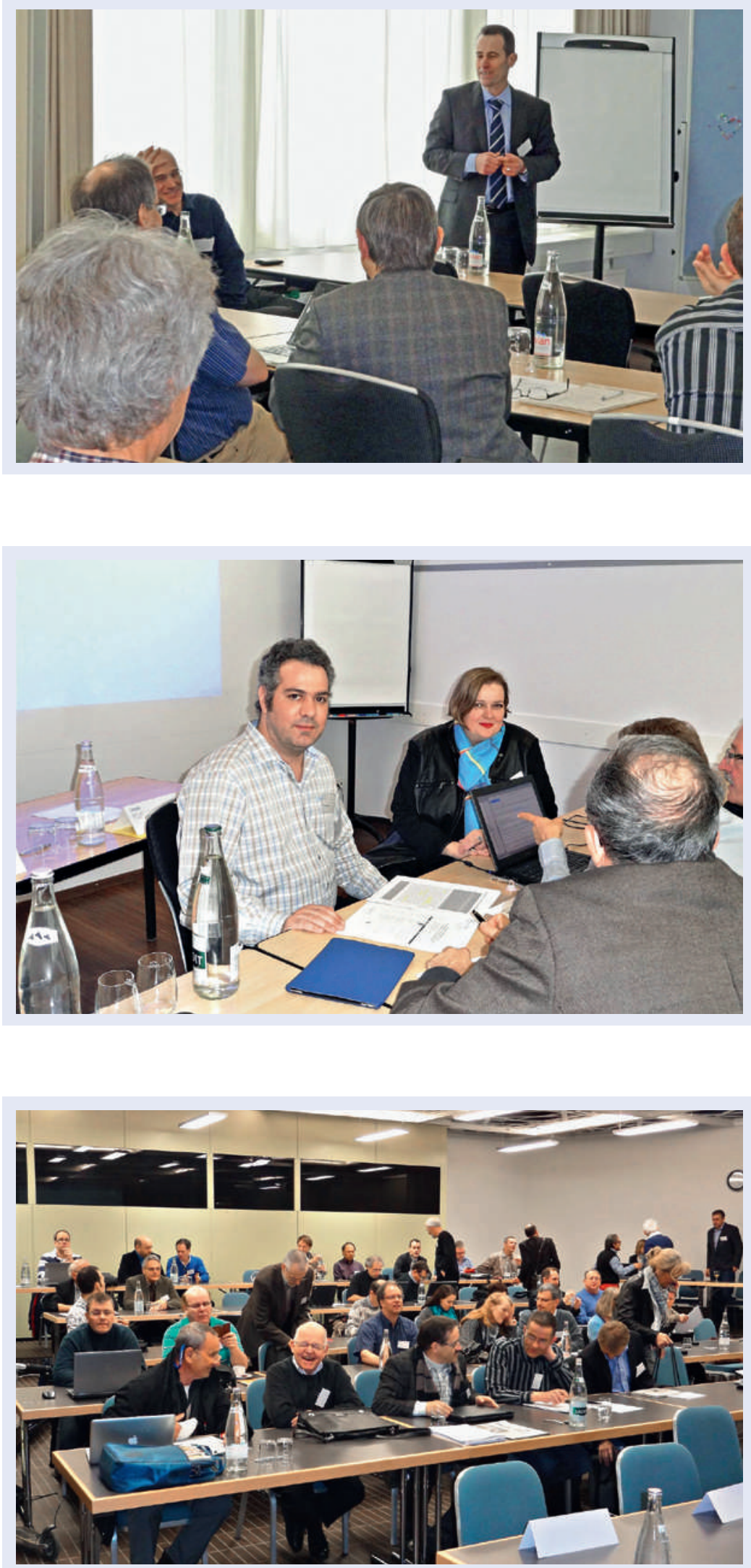

Workshop 2: Verlust der Datenparität - Ist das gefährlich?

Um Aussagen über Kosten im Gesundheitswesen tätigen zu können, sind aussagekräftige Daten der Leistungserbringer nötig. Was kann die Ärzteschaft dazu beitragen, die Datenqualtität und -quantität zu verbessern?

\section{Dr. Renato Laffranchi, Dr. Urs Stoffel:}

«Die Ärzteschaft braucht hochwertige Daten, damit sie die anstehenden Probleme, wie z.B. Verhandlung neuer Tarifmodelle (LeiKoV), Tarifstrukturrevisionen (TARVISION) und eine neue Grundlage für WZW-Verfahren, aktiv mitgestalten kann. Die Datenqualität ist heute ausgezeichnet, das ist primär darauf zurückzuführen, dass alle an der nationalen Datensammlung angeschlossenen Partner mit dem gleichen System arbeiten. Diesen entscheidenden Vorteil sollten wir auch in Zukunft aufrechterhalten. Die Datenquantität kann gesteigert werden, wenn sich noch mehr Ärzte entscheiden, an der nationalen Datensammlung teilzunehmen, und sich deshalb einem TrustCenter anschliessen.»

\section{Workshop 3: Heikle Fälle im TARMED}

Die Durchführung des Workshop rHeikle Fälle im TARMED, ist Tradition am Tarifdelegiertentag - Warum erfreut sich der Workshop so grosser Beliebtheit?

\section{Dr. Susanne Christen, Thomas Kessler:}

«Der Workshop hilft dem Tarifanwender bei der korrekten und wirtschaftlichen Leistungserfassung - im Speziellen auch bei komplexen Fällen, in denen die Tarifanwendung nicht immer eindeutig ist. Diesen Erfahrungs- und Wissensaustausch schätzen die Teilnehmer dieses Workshops sehr und er wird als hilfreich für den Praxisalltag erlebt.»
Der Tarifdelegiertentag ist mittlerweile zu einer festen Institution im Kalender der Delegierten geworden. Diese nutzen die angebotene Plattform für Information, Diskussion und Austausch. Das Ressort FMH Ambulante Tarife und Verträge Schweiz bedankt sich bei allen Referenten und Teilnehmern für die grosse Unterstützung und aktive Mitgestaltung.

Alle Referate und Publikationen finden Sie auf unserer Webseite www.fmh.ch $\rightarrow$ Tarmed $\rightarrow$ Publikationen $\rightarrow$ Tarifdelegiertentag.

Der nächste Tarifdelegiertentag findet am Mittwoch, 23.10.2013, im Hotel Bern statt.

Korrespondenz: FMH Ressort Ambulante Tarife und Verträge, Froburgstrasse 15, CH-4600 Olten, Tel. 03135912 30, tarife.ambulant[at]fmh.ch 